\title{
ISOLATED CHAIN RECURRENT POINTS FOR ONE-DIMENSIONAL MAPS
}

\author{
LOUIS BLOCK AND JOHN E. FRANKE
}

\begin{abstract}
For a continuous map of the interval to itself or of the circle to itself, we show that any isolated chain recurrent point is eventually periodic. Furthermore, an isolated chain recurrent point which is not in the orbit of a critical point and has no critical point in its orbit is periodic.
\end{abstract}

This paper is concerned with isolated chain recurrent points (i.e. chain recurrent points which are isolated in the chain recurrent set) for continuous maps of the interval or the circle. Our first result is the following.

THEOREM A. Let $f$ be a continuous map of the compact interval to itself or of the circle to itself. Then any isolated chain recurrent point of $f$ is eventually periodic.

Note that Theorem A becomes false if one replaces isolated chain recurrent point by isolated nonwandering point, as the example of Coven and Nitecki in $\S 4$ of [5] shows. In this example there is an isolated nonwandering point $x_{0}$ with an infinite orbit. Theorem A implies that $x_{0}$ cannot be isolated in the chain recurrent set.

For the simplest maps, the Morse-Smale homeomorphisms [6], the chain recurrent set consists of a finite set of periodic points. However, the examples in $\S 4$ of [1] and $\S 3$ of [7] show that an isolated chain recurrent point need not be periodic. In each of these examples the isolated (nonperiodic) chain recurrent point is in the orbit of a critical point.

We construct a slightly different type of example. Let $g$ be the map of the interval $[0,1]$ to itself with $g(0)=0, g(1 / 4)=1 / 2, g(3 / 10)=13 / 40, g(2 / 5)=3 / 8$, $g(1 / 2)=1 / 2, g(3 / 4)=7 / 8$ and $g(1)=1$ such that $g$ is linear on each of the intervals $[0,1 / 4],[1 / 4,3 / 10],[3 / 10,2 / 5],[2 / 5,1 / 2],[1 / 2,3 / 4]$ and $[3 / 4,1]$. Let $f$ be the map of the circle to itself obtained from $g$ by identifying 0 and 1 . Then $f$ has three fixed points, $1 / 2$ which is a source or expanding fixed point, $7 / 20$ which is a sink or contracting fixed point, and $0=1$ which is expanding on the "right" and contracting on the "left". It is easy to verify that the chain recurrent set consists of the sink $7 / 20$, the interval $[1 / 2,1]$ and the points $1 / 4,1 / 8,1 / 16, \ldots$. In fact, all other points are in the stable manifold of the sink $7 / 20$. Thus, $f$ has infinitely many isolated chain recurrent points which are not periodic (and also not homoclinic [2, 3]). Note that each of these isolated chain recurrent points has a critical point in its orbit (namely 1/4).

Thus, we see that an isolated chain recurrent point $x$ need not be periodic if either $x$ is in the orbit of a critical point or there is a critical point in the orbit of $x$. Our second theorem shows that except for these situations $x$ must be periodic.

Received by the editors June 16, 1984 and, in revised form, September 17, 1984 .

1980 Mathematics Subject Classification. Primary 54H20. 
THEOREM B. Let $f$ be a continuous map of the compact interval to itself or of the circle to itself, and let $x$ be an isolated chain recurrent point of $f$. Suppose that $x$ is not in the orbit of a critical point, and no critical point is in the orbit of $x$. Then $x$ is periodic.

We will let $f$ denote a continuous map of the circle $S^{1}$ to itself, and let $d$ denote a metric on $S^{1}$. The reader can easily see that the proof of the theorem works as well if $f$ is a continuous map of the interval to itself.

Let $x$ and $y$ be points on $S^{1}$. An $\varepsilon$-chain from $x$ to $y$ is a finite sequence of points $\left\{x_{0}, x_{1}, \ldots, x_{n}\right\}$ of $S^{1}$ with $x=x_{0}, y=x_{n}$, and $d\left(f\left(x_{i-1}\right), x_{i}\right)<\varepsilon$ for $i=1, \ldots, n$. We say $x$ can be chained to $y$ if for every $\varepsilon>0$ there is an $\varepsilon$-chain from $x$ to $y$, and we say $x$ is chain recurrent if $x$ can be chained to $x$. The set of chain recurrent points is denoted $R(f)$. We let $R_{\varepsilon}(x)$ denote the set of $y \in S^{1}$ such that there is an $\varepsilon$-chain from $x$ to $y$ and $\omega(x)$ denote the set of limit points of the orbit of $x$ (precisely, $z \in \omega(x)$ if and only if some subsequence of the sequence $\left(f^{n}(x)\right.$ ) converges to $z$ ). We say $x$ is eventually periodic if some element of the orbit of $x$ is periodic, and we say $x$ is a critical point of $f$ if $f$ is not a local homeomorphism at $x$.

The following three lemmas are easily verified. Lemma 2 is proved in [4]. Lemmas 2 and 3 hold on any compact metric space.

LEMMA 1. For any $x \in S^{1}$ and any $\varepsilon>0, R_{\varepsilon}(x)$ has a finite number of components. Each component is an open interval which contains an element of the orbit of $x$.

LEMMA 2. Let $x \in S^{1}$. If $x \in R(f)$, then for each positive integer $k, f^{k}(x)$ can be chained to $x$.

LEMMA 3. If $x \in R(f)$ and $z \in \omega(x)$, then $z$ can be chained to $x$.

The heart of the proof of Theorem A is contained in the next two lemmas.

LEMMA 4. Let $x \in R(f)$ and suppose that $x$ is an endpoint of an open interval $J \subset S^{1} \backslash R(f)$ such that $x$ can be chained to each point of $J$. Then $x$ is eventually periodic.

Proof. Suppose $x$ is not eventually periodic, and let $z \in \omega(x)$. By Lemma 3, $z$ can be chained to each point of $J$. Note that none of the intervals $J, f(J), f^{2}(J), \ldots$ can contain a point in the orbit of $x$, since this would force a point in $J$ to be chain recurrent (using Lemma 2). It follows that $z \in \omega(y)$ for each $y \in J$. Thus, each point of $J$ is chain recurrent, a contradiction. Q.E.D.

LEMMA 5. Let $x \in R(f)$. Then either $x \in \omega(x)$ or $x$ is an endpoint of an open interval $J$ such that $x$ can be chained to each point of $J$.

Proof. Assume $x \notin \omega(x)$, and let $z \in \omega(x)$. Note that $x \notin \omega(z)$, for this would imply $x \in \omega(x)$. Similarly, $x$ is not in the orbit of $z$. Thus, if $\delta$ denotes the distance from $x$ to the orbit of $z$, then $\delta>0$.

Let $n$ be a positive integer. By Lemma $1, R_{1 / n}(z)$ has a finite number of components, each of which contains a point in the orbit of $z$. It follows from Lemma 3 that $x$ is in one of these components, which we call $J_{n}$. Note that the length of $J_{n}$ is at least $\delta$ and $J_{n+1} \subset J_{n}$.

Let $K=\bigcap_{n=1}^{\infty} J_{n}$. Then $z$ (and hence $x$ ) can be chained to every point in $K$, and $K$ contains an open interval $J$ with endpoint $x$. Q.E.D. 
PROOF OF THEOREM A. Suppose that $x$ is an isolated chain recurrent point of $f$. We may assume that $x$ is not periodic (or else we are done). It follows that $x \notin \omega(x)$, and thus, Lemma 5 implies that $x$ satisfies the hypothesis of Lemma 4 . Hence, $x$ is eventually periodic.

We begin the proof of Theorem B with the following lemma. Note that the proof is valid on any compact metric space.

LEMMA 6. Suppose $f(p)=p$ and $p$ can be chained to $x$ where $x \neq p$. There is a sequence $\left(z_{i}\right)$ approaching $p$ such that for each $i, z_{i} \neq p$ and $z_{i}$ can be chained to $x$.

ProOF. Let $\delta>0$. For each $\gamma>0$ set $U(\gamma)=\left\{y \in S^{1}: d(y, p)<\gamma\right\}$ and $V(\gamma)=\left\{y \in S^{1}: d(y, z)<\gamma\right.$ for some $\left.z \in f(U(\gamma))\right\}$. Choose $\gamma$ small enough that $x \notin U(\gamma) \cup V(\gamma)$ and if $y \in U(\gamma) \cup V(\gamma)$, then $d(y, p)<\delta$. For each $k>1 / \gamma$ there is a point $s_{k}$ of a $(1 / k)$-chain from $p$ to $x$ with $s_{k} \in V(\gamma) \backslash U(\gamma)$. If $z$ is a limit point of the sequence $\left(s_{k}\right)$, then $z \neq p, d(z, p) \leq \delta$, and $z$ can be chained to $x$. Since $\delta$ was arbitrary, the conclusion of the lemma follows. Q.E.D.

PROOF OF THEOREM B. Suppose that $x$ is not periodic. By Theorem A, $x$ is eventually periodic. Hence, replacing $f$ by an iterate of $f$ if necessary (using the fact that $R(f)=R\left(f^{k}\right)$ ), we may assume that $f(x)=p$, where $p$ is a fixed point of $f$ and $x \neq p$.

Let $R(p)=\left\{y \in S^{\mathbf{1}}: p\right.$ can be chained to $\left.y\right\}$. It is easy to verify that $R(p)$ is closed and $f(R(p))=R(p)$. Furthermore, since $f(p)=p$, it follows from Lemma 1 that $R(p)$ is a closed interval. By Lemma $2, x \in R(p)$. If $R(p)$ did not contain a neighborhood of $x$, then $x$ would be an endpoint of $R(p)$. Thus, $x$ would be either periodic (of period 1 or 2 ) or in the orbit of a critical point. In either case, we obtain a contradiction, so $R(p)$ contains a neighborhood of $x$.

Let $V$ be a neighborhood of $x$ such that $f$ is a homeomorphism on $V$ and $V \subset$ $R(p)$. By Lemma 6 , there is a sequence $\left(z_{i}\right)$ approaching $p$ such that for each $i$, $z_{i} \neq p$ and $z_{i}$ can be chained to $x$. We may assume that $z_{i} \in f(V)$ for each $i$. Thus, for each $i$ there is a unique $y_{i} \in V$ with $f\left(y_{i}\right)=z_{i}$. The sequence $\left(y_{i}\right)$ approaches $x$, and each $y_{i}$ is chain recurrent. This contradicts our hypothesis that $x$ is an isolated chain recurrent point.

\section{REFERENCES}

1. L. Block, Diffeomorphisms obtained from endomorphisms, Trans. Amer. Math. Soc. 214 (1975), 403-413.

2. __ Homoclinic points of mappings of the interval, Proc. Amer. Math. Soc. 72 (1978), 576-580.

3. L. Block, E. M. Coven, I. Mulvey and Z. Nitecki, Homoclinic and nomuandering points for maps of the circle, Ergodic Theory Dynamical Systems 3 (1983), 521-532.

4. L. Block and J. Franke, The chain recurrent set for maps of the interval, Proc. Amer. Math. Soc. 87 (1983), 723-727.

5. E. M. Coven and Z. Nitecki, Nomwandering sets of the powers of maps of the interval, Ergodic Theory Dynamical Systems 1 (1981), 9-31.

6. Z. Nitecki, Differentiable dymamics: an introduction to the orbit structure of diffeomorphisms, MIT Press, Cambridge, Mass., 1971.

7. L. S. Young, A closing lemma on the interval, Invent. Math. 54 (1979), 179-187.

Department of Mathematics, University of Florida, Gainesville, Florida 32611

Department of Mathematics, North Carolina State University, Raleigh, NORTH CAROLINA 27695 